Communications in Physics, Vol. 25, No. 4 (2015), pp. 299-308

DOI:10.15625/0868-3166/25/4/7676

\title{
GRAVITATIONAL WAVEFORMS FROM MULTIPLE-ORBIT SIMULATIONS OF BINARY NEUTRON STARS
}

\author{
NGUYEN QUYNH LAN
}

Hanoi National University of Education, 136 Xuan Thuy, Cau Giay

and

Joint Institute for Nuclear Astrophysics (JINA), University of Notre Dame, Notre Dame, Indiana 46556, USA

\section{IN-SAENG SUH}

Center for Astrophysics, Department of Physics and Center for Research Computing, University of Notre Dame, Notre Dame, Indiana 46556, USA

GRANT J. MATHEWS AND J. REESE HAYWOOD

Center for Astrophysics, Department of Physics, University of Notre Dame, Notre Dame, Indiana 46556, USA

E-mail: nquynhlan@hnue.edu.vn

Received 26 October 2015

Accepted for publication 12 December 2014

\begin{abstract}
We study the gravitational wave emission of equal-mass neutron stars in binary orbits as the stars approach the inner most last stable circular orbit. We illustrate the extraction of gravitational wave forms in a sequence of quasi-circular orbit simulations including the general relativistic hydrodynamic response of the stars. We compare the computed results with the Newtonian and post Newtonian results and show that substantial differences can arise as the orbits approach the final inspiral.
\end{abstract}

Keywords: binary neutron stars, numerical relativity, ADM.

\section{INTRODUCTION}

Current interferemetric gravity wave observatories such as LIGO [1], GEO600 [2], GEOHF [3, 4], TAMA300 [5] and VIRGO [6] have been taking data for some time [7-10], while a number of second generation observatories such as Advanced LIGO [11], Advanced VIRGO [12] and KAGRA [13] soon will be online. These observatories seek to detect gravity-wave emission from various sources, e.g. from core collapse supernovae, neutron star orbits, the stochastic cosmic background, etc. [1]. Of the many systems that emit gravitational waves, compact neutron-star and/or black-hole binaries are thought to be the best candidates for detecting gravitational radiation [14]. Indeed, the first discovery of a black hole - black hole merger has been reported. The number (C)2015 Vietnam Academy of Science and Technology 
of neutron star - neutron star systems detectable by Advanced LIGO [15] is estimated [14, 16-21] to be of order several events per year based upon observed close binary-pulsar systems [22,23].

To date there have been numerous attempts to calculate theoretical templates for gravity waves from compact binaries based upon numerical and/or analytic approaches (see for example [24-33]). However, most approaches utilize a combination of Post-Newtonian (PN) techniques supplemented with quasi-circular orbit calculations and then applying full GR for only the last few orbits before inspiral. In a previous paper [34] we have reported on a general relativistic hydrodynamics approach that can compute many orbits stably and efficiently from the PN regime until the last stable orbits without the need to invoke the quasi-circular orbit condition. We established the numerical stability of this approach based upon many orbit simulations of quasi-circular orbits and showed that this approach is straightforwardly scalable to evolve to the $\sim 10^{4}$ orbits within the LIGO frequency range. In this paper we present a first study of associated gravity waveforms from such multiple orbit simulations.

When binary neutron stars are well separated, the Post-Newtonian (PN) approximation is sufficiently accurate [35]. In the PN scheme, the stars are often treated as point masses, either with or without spin. At third order, for example, it has been estimated [36-38] that the errors due to assuming the stars are point masses is less than one orbital rotation [36] over the $\sim 16,000$ cycles that pass through the LIGO detector frequency band [14]. Nevertheless, it has been noted in many works $[32,39-50]$ that relativistic hydrodynamic effects might be evident even at the separations $(\sim 10-100 \mathrm{~km})$ relevant to the LIGO window.

Indeed, the templates generated by PN approximations, unless carried out to fifth and sixth order [36,37], may not be accurate unless the finite size and proper fluid motion of the stars is taken into account. In essence, the signal emitted during the last phases of inspiral depends on the finite size and equation of state (EoS) through the tidal deformation of the neutron stars and the cut-off frequency when tidal disruption occurs.

Numeric and analytic simulations [51-59] of binary neutron stars have analyzed the approach to the innermost stable circular orbit (ISCO). While these simulations represent some of the most accurate to date, many simulations have only followed the evolution for a handful of orbits and are based upon an extrapolation of quasi-circular orbits. With $\sim 16,000$ cycles passing though the LIGO frequency band, it may questionable whether templates based on only a small number of orbits are sufficiently accurate to describe the full evolution of the system. Moreover, although one can obtain a solution to Einstein equations in the quasi-circular orbit condition, there is no guarantee that the true dynamical evolution actually passes through a given set quasi-circular solutions.

Accurate templates may eventually require the ability to calculate many orbits, including the radiation back reaction and relativistic hydrodynamic effects. Ideally, one would like to calculate from the post-Newtonian regime to near the inner most stable circular orbit (ISCO).

Toward that end, we have developed an approach [34] based upon the general relativistic hydrodynamics formalism developed in $[40,42,60]$ that can evolve from the post-Newtonian to ISCO regimes in a single calculation. We showed [34] that it is straightforwardly scalable to the computation of the continuous evolution through the $\sim 10^{4}$ orbits in the LIGO window. Here, we illustrate an application of the formalism to estimate of the emergent gravity wave signal. The method for including the radiation back reaction and the extraction of the outgoing gravity 
wave template is described in Section 2 along with an illustrative extraction of the gravity wave parameters. Conclusions are presented in Sections 3.

\section{GRAVITATIONAL WAVES}

The physical processes occurring during the last orbits of a neutron star binary are currently a subject of intense interest. As the stars approach their final orbits it is expected that the coupling of the orbital motion to the hydrodynamic evolution of the stars in the strong relativistic fields could provide insight into various physical properties of the coalescing system [34, 45, 61]. In this regard, careful modeling is needed which includes both the nonlinear general relativistic and hydrodynamic effects as well as a realistic neutron star equation of state.

Because our method of solving the field equations [34,40,60] does not explicitly evolve gravitational radiation we use a multipole expansion originally developed in [62] as described in $[40,60]$. The gravitational radiation signal is derived via a multipole expansion of the metric perturbation to the hexadecapole $(l=4)$ order including both mass and current moments and a correction for the slow motion approximation. In [34], we computed quasi-equilibrium circular orbit conditions for two neutron stars initially of gravitational mass $1.44 \mathrm{M}_{\odot}$. We then considered configurations as a function of total angular momentum from the post-Newtonian regime up to the point that the stars enter the inspiral phase [34]. In this paper we perform an illustrative calculation of the angular momentum and power loss rate and reconstruct the gravitational wave form. We also summarize how to infer the possible signal to noise in the LIGO sensitivity band.

In general it is possible to express the emission of gravitational radiation in terms of an "exact" expansion of multipole moments of the effective stress energy tensor, including corrections for the so-called "slow motion" approximation [62]. It is important to appreciate that these formulae can apply to strong-field sources as well as to weak field sources [62] as long as the relevant components of the effective stress energy tensor can be identified.

Since in this paper, we are only concerned with orbital motion of equal mass binaries, the multipole expansions reduce to only a few nonzero terms. These we evaluate and test for convergence of the expansion.

In any asymptotically flat coordinate system (such as the one we are using here) in which the gravity waves far from the source can be characterized as linear metric perturbations propagating on a flat background, the transverse traceless part of the metric perturbation characterizes the radiation completely. This metric perturbation then can be expressed $[40,60,62]$ in terms of the mass-multipole $\left(I^{l m}\right)$ and current-multipole $\left(S^{l m}\right)$ moments as

$$
h_{j k}^{T T}=\sum_{l=2}^{\infty} \sum_{m=-l}^{l}\left[r^{-1(l)} I^{l m}(t-r) T_{j k}^{E 2, l m}+r^{-1(l)} S^{l m}(t-r) T_{j k}^{B 2, l m}\right],
$$

where the superscript $T T$ denotes the transverse traceless part of the metric perturbation and the notation ${ }^{(l)} I^{l m}$ and ${ }^{(l)} S^{l m}$ denotes the $l^{t h}$ time derivative of the respective moments. As usual, the gravitational wave strain can be given in terms of two polarization $h_{\times}$and $h_{+}$.

From this, the general expression for energy loss is

$$
\frac{d E}{d t}=\frac{1}{32 \pi} \sum_{l=2}^{\infty} \sum_{m=-l}^{l}\left\langle\left.\left.\right|^{(l+1)} I^{l m}\right|^{2}+\left|{ }^{(l+1)} S^{l m}\right|^{2}\right\rangle,
$$


where the brackets denote averages over several wavelengths. Angular momentum loss can similarly be written

$$
\begin{aligned}
\frac{d J}{d t}=\quad & \frac{i}{32 \pi} \sum_{l=2}^{\infty} \sum_{m=-l}^{l}\left\langle{ }^{(l)} I^{l m *} m^{(l+1)} I^{l m}\right\rangle \\
& +\left\langle{ }^{(l)} S^{l m *} m^{(l+1)} S^{l m}\right\rangle,
\end{aligned}
$$

where the expression in Eq. (3) assumes an alignment of the angular momentum vector with the $\mathrm{z}$ axis.

The radiation reaction potential for the loss of orbital momentum can also be written [40] in terms of these moments.

$$
\chi=\frac{1}{32 \pi} \sum_{l=2}^{\infty} \sum_{m=-l}^{l} x_{i} x_{j}\left\langle\left.\left.\right|^{(l+1)} I^{l m}\right|^{2}+\left.\left.\right|^{(l+1)} S^{l m}\right|^{2}\right\rangle,
$$

The derivation of the relevant mass and current moments in our coordinates is straightforward as described in detail in Refs. [40,60] and need not be repeated here. The contribution from both the current moments and slow-motion correction is expected to be small. We compute terms out to $\omega^{10}$, which includes mass multipoles out to $l=4$, current multipoles out to $l=3$ and the leading correction for the slow motion correction.

In Table 1 we summarize the relative contributions of various moments to the energy and angular momentum loss rates for a few values of total angular momentum $J$ based upon the fiducial multiple orbit simulation or Ref. [34] utilizing the MW equation of state for two equal mass neutron stars of gravitational mass $M_{g}=1.44 \mathrm{M}_{\odot}$. The orbit parameters for various fixed angular momenta are summarized in Table 2 from Ref. [34]. As expected, the quadrupole term dominates by more than an order of magnitude. The next largest term is the slow motion correction which contributes only a few percent to the gravitational radiation and tends to decrease the loss rate. Hence, we conclude that the moment expansion indeed converges quickly.

Table 1. Contribution of various moments to energy and angular momentum loss rates

\begin{tabular}{cccccc}
\hline \hline$J$ & $\dot{E}$ & $l=2, m=2$ & Slow Motion Correction & $l=4, m=2$ & $l=4, m=4$ \\
\hline $2.7 \times 10^{11}$ & $5.06 \times 10^{-9}$ & $2.63 \times 10^{-9}$ & $-9.86 \times 10^{-11}$ & $5.56 \times 10^{-17}$ & $1.02 \times 10^{-13}$ \\
$2.8 \times 10^{11}$ & $3.57 \times 10^{-9}$ & $1.85 \times 10^{-9}$ & $-6.76 \times 10^{-11}$ & $3.51 \times 10^{-17}$ & $6.32 \times 10^{-14}$ \\
$3.0 \times 10^{11}$ & $1.72 \times 10^{-9}$ & $8.90 \times 10^{-10}$ & $-3.08 \times 10^{-11}$ & $1.29 \times 10^{-17}$ & $2.32 \times 10^{-14}$ \\
\hline$J$ & $j$ & $l=2, m=2$ & Slow Motion Correction & $l=4, m=2$ & $l=4, m=4$ \\
\hline $2.7 \times 10^{11}$ & $1.26 \times 10^{-1}$ & $1.17 \times 10^{-1}$ & $-4.40 \times 10^{-3}$ & $2.52 \times 10^{-9}$ & $4.53 \times 10^{-6}$ \\
$2.8 \times 10^{11}$ & $1.77 \times 10^{-1}$ & $9.20 \times 10^{-2}$ & $-3.36 \times 10^{-3}$ & $1.75 \times 10^{-9}$ & $3.14 \times 10^{-6}$ \\
$3.0 \times 10^{11}$ & $1.07 \times 10^{-1}$ & $5.53 \times 10^{-2}$ & $-1.92 \times 10^{-3}$ & $8.04 \times 10^{-10}$ & $1.45 \times 10^{-6}$ \\
\hline
\end{tabular}


Table 2. Orbital parameters for each EoS

\begin{tabular}{ccccccc}
\hline \hline EoS & $J\left(\mathrm{~cm}^{2}\right)$ & $\omega\left(\mathrm{rad} \mathrm{s}^{-1}\right)$ & $d_{p}(\mathrm{~km})$ & $d_{c}(\mathrm{~km})$ & $M_{A D M}\left(M_{\odot}\right)$ & $\rho_{c}\left(\mathrm{~g} \mathrm{~cm}^{-3}\right)$ \\
\hline MW & $2.6 \times 10^{11}$ & 780.92 & 65.22 & 51.52 & 1.391 & $1.67 \times 10^{15}$ \\
& $2.7 \times 10^{11}$ & 671.85 & 71.18 & 57.24 & 1.393 & $1.62 \times 10^{15}$ \\
& $2.8 \times 10^{11}$ & 602.80 & 76.94 & 61.86 & 1.394 & $1.60 \times 10^{15}$ \\
& $3.0 \times 10^{11}$ & 482.30 & 86.91 & 72.36 & 1.396 & $1.55 \times 10^{15}$ \\
& $3.5 \times 10^{11}$ & 300.46 & 116.13 & 100.8 & 1.399 & $1.44 \times 10^{15}$ \\
$3.8 \times 10^{11}$ & 235.72 & 136.93 & 119.74 & 1.401 & $1.39 \times 10^{15}$ \\
\hline
\end{tabular}

The sensitivity of the gravity wave frequency to the equation of state was summarized in Ref. [34]. Here we analyze the gravity wave characteristics based upon one representative equation of state. Fig. 1 shows $f, h, \dot{E}, \dot{J}$ as a function of time to inspiral for a simulation based upon the fiducial MW EoS. For this plot we adopt $h \equiv\left(h_{+}+h_{\times}\right) / 2$. In Fig. 2 we show various parameters, i.e. $f, h, \dot{E}, \dot{J}$ characterizing the gravity wave signals as a function of total angular momentum $J$ for calculations based upon the MW equation of state of Ref. [68]. In this figure, the points are the numerical results. The lines drawn are polynomial fits to these computational results. These analytic functions are summarized in Table 3.

Table 3. Polynomial fits to $f, h, \dot{E}, \dot{J}$

\begin{tabular}{c|ccccc}
\hline \hline & $a_{0}$ & $a_{1} x$ & $a_{2} x^{2}$ & $a_{3} x^{3}$ & $a_{4} x^{4}$ \\
\hline$f$ & 42.3 & -32.65 & 8.77 & -0.802 & \\
$h$ & 44.01 & $-29.48 \mathrm{E}+02$ & 7.221 & -0.6144 & \\
$\dot{E}$ & 3752.90 & -4488.11 & 2009.89 & -399.21 & 29.66 \\
$\dot{J}$ & 14.14 & -12.00 & 3.42 & -0.325 & \\
\hline
\end{tabular}

Having analytic fits to the various parameters as a function of $J$ we can convert them to time in the limit that only gravity waves affect the orbit decay time by integrating the angular momentum loss timescale, i.e.

$$
t=\int_{J_{\text {coll }}}^{J} \frac{d J^{\prime}}{\dot{J}}
$$

where $J_{\text {coll }}$ is the minimum $J$ value before collapse of the orbit. 


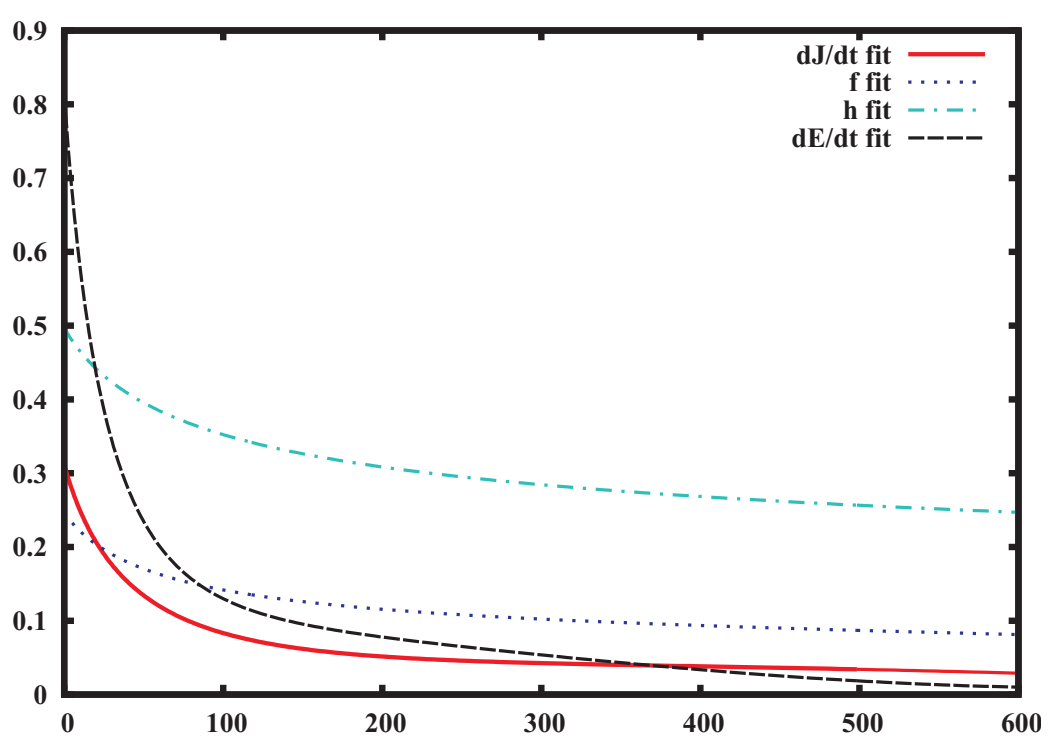

Fig. 1. Plot of $\mathrm{f}, \mathrm{h}, \dot{E}$, and $\dot{J}$ versus time to inspiral

For comparison the expected increase in gravity wave frequency from the post-Newtonian and Newtonian estimate is compared with our calculations on Fig. 3. We see the chirp as the stars approach. The fact that there is less increase in frequency as the stars approach implies that there are more cycles per time bin so that the inspiral may be easier to detect [69].

Ultimately, however, one wishes to know the actual signal to noise response in an gravity wave detector. The detector response in real applications involves a fourier transform integrated over many orbits within the LIGO frequency band. To estimate of the effect of these orbits on the detector signal to noise one must simply fourier transform the observed time-dependent gravity wave form given above. That is, the time dependent detector strain is given by,

$$
h_{t}(t)=A \times h \cos \int 2 \pi f d t,
$$

where $A$ is a parameter that depends upon detector inclination and response, while $h$ is defined above and given in Fig. 1. The detailed time evolution of $h_{\times}$and $h_{+}$are shown in Fig. 4 and Fig. 5 for the last $200 \mathrm{~ms}$ of inspiral.

The fourier transform is then written,

$$
\tilde{h}(f)=\int e^{2 \pi f t} h_{t}(t) d t
$$

The signal to noise per unit frequency is then,

$$
\frac{d(S / N)^{2}}{d f}=\frac{4\left|\tilde{H}^{2}\right|}{S_{n}}
$$

where $S_{n}$ is the detector spectral density function. For our purposes $S_{n}$ can be taken from the analytic approximations of Ref. [70]. 


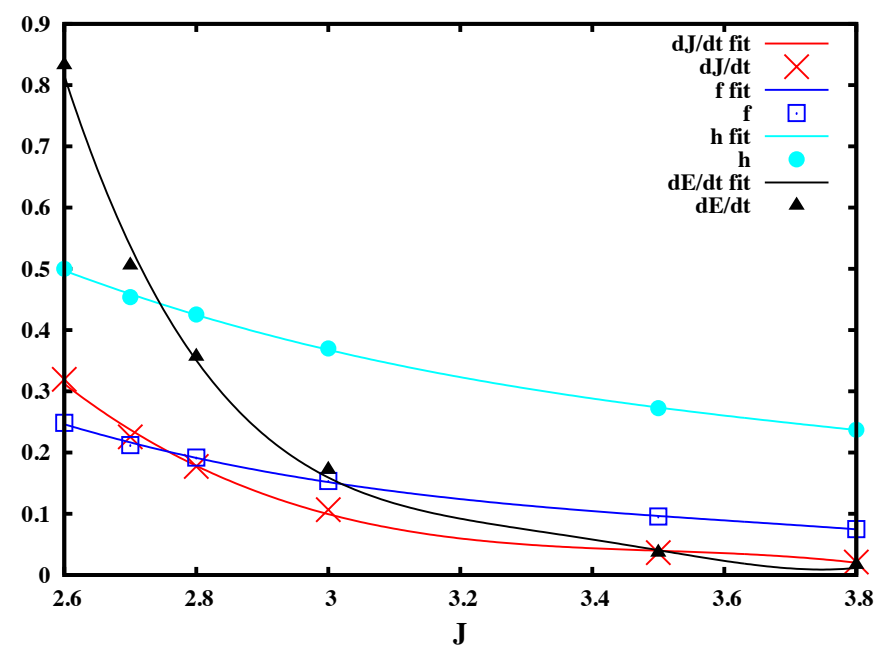

Fig. 2. Plot of $f, h, \dot{E}$, and $\dot{J}$ versus $J$. The points are calculated. The lines are the polynomial fits given in Table 3

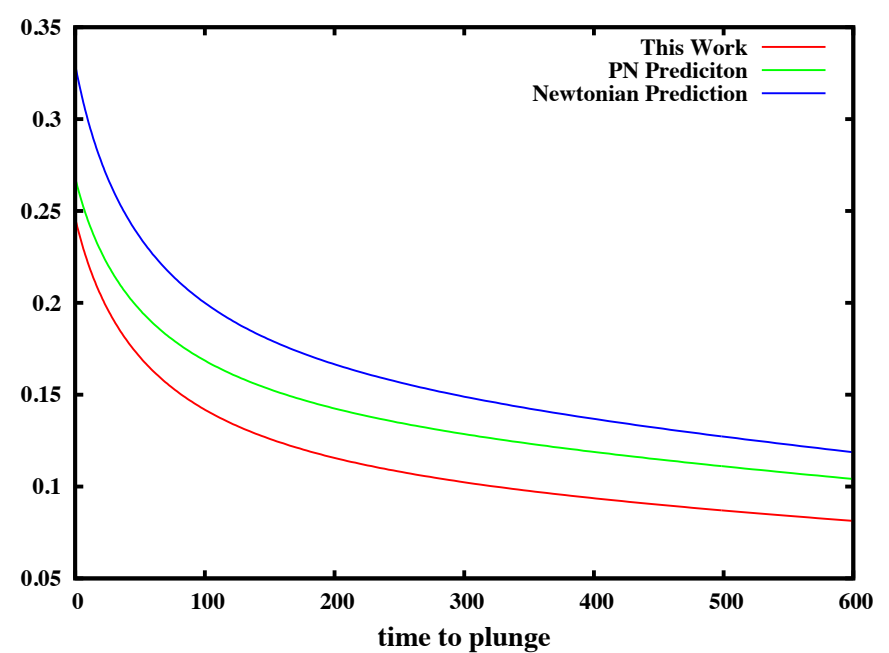

Fig. 3. Plot of gravity wave frequency $f$ versus time to inspiral from this work compared with the the PN prediction and the Newtonian prediction 


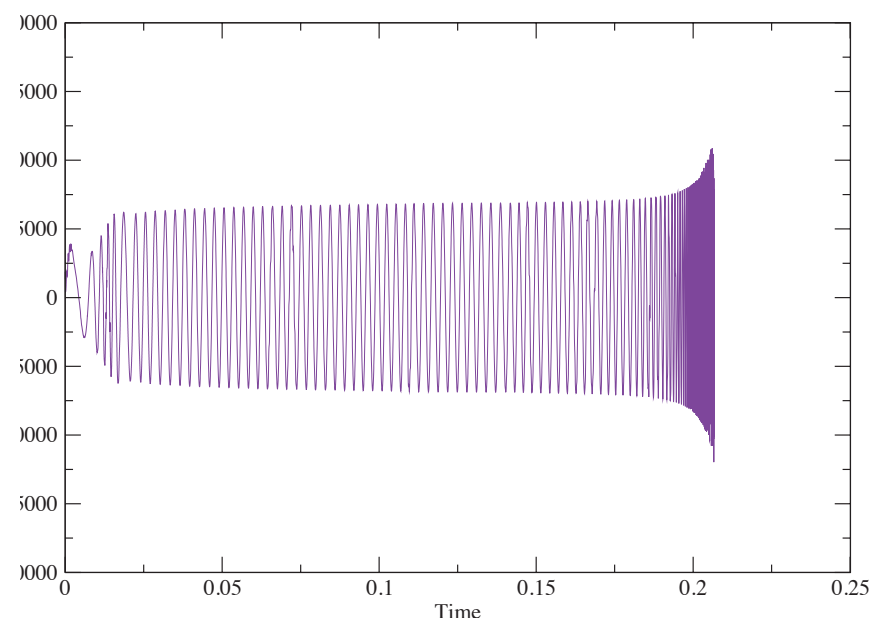

Fig. 4. Plot of the strain $h_{\times}$, versus time for the last $200 \mathrm{~ms}$ before inspiral.

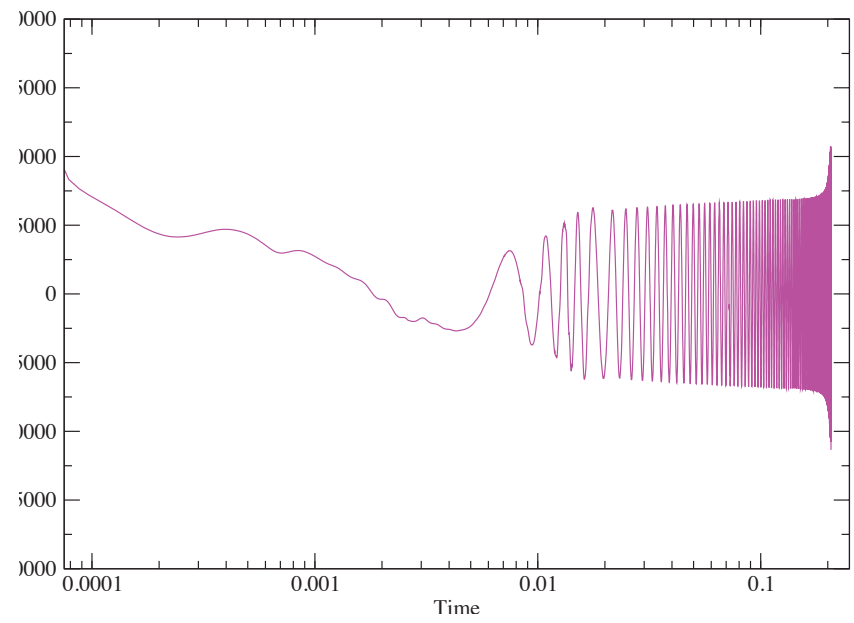

Fig. 5. Plot of the strain $h_{+}$, versus time for the last $200 \mathrm{~ms}$ before inspiral.

\section{CONCLUSIONS}

We have computed the emergent gravity wave signal from a multiple orbit simulation of an equal-mass binary neutron star system. The gravitational wave form was obtained via a multiple expansion up to $l=4$ and including the corrections to the slow motion approximation. Although our approach employs a number of approximations including a conformally flat metric, a multipole expansion, and quasi-stationary orbits, we have shown that this calculation can be performed efficiently with limited computer resources. Hence, we suggest that the templates developed by this method should be applied in current gravity wave observatories. 


\section{ACKNOWLEDGEMENTS}

Work at the University of Notre Dame (G.J.M.) supported by the U.S. Department of Energy under Nuclear Theory Grant DE-FG02-95-ER40934, and by the University of Notre Dame Center for Research Computing. One of the authors (N.Q.L.) was supported in part by the National Science Foundation through the Joint Institute for Nuclear Theory (JINA); Ministry of Education (MOE) Grant No. B2014-17-45.

This work was support by JSPS Core-to-Core Program, School fund of Institute for Cosmic Ray Research, University of Tokyo.

\section{REFERENCES}

[1] LIGO Scientific Collaboration, http://www.ligo.org

[2] GEO Collaboration, Geo600 http://www.geo600.uni-hannover.de

[3] Luck H, Affeldt C, Degallaix J, Freise A, Grote H, et al., J.Phys.Conf.Ser. 228 (2010) 012012

[4] Affeldt C, Danzmann K, Dooley K, Grote H, Hewitson M, et al., Class.Quant.Grav. 31 (2014) 224002

[5] TAMA300 Collaboration, Tama300 http://tamago.mtk.nao.ac.jp

[6] VIRGO Collaboration, Virgo http://wwwcascina.virgo.infn.it

[7] B. Abbott et al., Phys. Rev. D 69 (2004) 082004

[8] B. Abbott et al., Phys. Rev. D 69 (2004) 122004

[9] B. Abbott et al., Phys. Rev. D 73 (2006) 062001

[10] B. Abbott et al., Phys. Rev. D 73 (2006) 102002

[11] G. M. Harry (LIGO Scientific Collaboration) Class .Quant. Grav. 27 (2010) 084006

[12] F. Acernese et al. (VIRGO) Class. Quant. Grav. 32 (2015) 024001

[13] K. Somiya (KAGRA Collaboration) Class. Quant. Grav. 29 (2012) 124007

[14] K S. Thorne Compact Stars in Binaries, J. van Paradijs, E. P. J. van den Heuvel, and E. Kuulkers, editors, IAU Symp. 165 (1996) 153

[15] G. M. Harry Class. Quant. Grav. 27 (2010) 084006

[16] F. A. Rasio and S. L. Shapiro Class. Quant. Grav. 16 (1999) 1

[17] M. Bailes Compact Stars in Binaries, J. van Paradijs, E. P. J. van den Heuvel, and E. Kuulkers, editors, IAU Symp. 165 (1996) 213

[18] A. V. Tutukov and L. R. IungelSon MNRAS 260 (1993) 675

[19] E. S. Phinney Astrophys J. 380 L17 (1991)

[20] K. Kalogera et al., Astrophys. J. Lett, 614, L137 (2014)

[21] J. Abadie et al. Class. Quant. Grav. 27 (2014) 173001

[22] M. Burgay et al. Nature 426, (2003) 531

[23] J. M. Lattimer Ann. Rev. Nucl Part. Sci 62 ( 2012) 485

[24] T. A. Apostolatos Phys. Rev. D 54, (1996) 2421

[25] S. Droz and E. Poisson Phys. Rev. D 56, ( 1997) 4449

[26] B. S. Sathyaprakash Class. Quant. Grav. 17 L157 ( 2000)

[27] A. Buonanno, Y. Chen, and M. Vallisneri Phys. Rev. D 67 (2003) 024016

[28] S. Bose Phys. Rev. D 71 (2005) 082001

[29] C. D. Ott, A. Burrows, L. Dessart, and E. Livne Phys. Rev. Lett. 96 ( 2006) 201102

[30] P. Ajith, N. Fotopoulos, S. Privitera, A. Neunzert, N. Mazumder and A. Weinstein Phys. Rev. D 89 ( 2014 ) 084041

[31] F. Pannarale, E. Berti, K. Kyutoku, B. D. Lackey, and M. Shibata Phys. Rev. D 92 ( 2015) 081504

[32] M. Agathos, J. Meidam, W. Del Pozzo, T. G. F. Li, M. Tompitak, J. Veitch, S. Vitale, and C. Van Den Broeck Phys. Rev. D 92 (2015) 023012

[33] J. A. Clark, A. Bauswein, N. Stergioulas, and D. Shoemaker, eprint arXiv:1509.08522, 2015

[34] I-S. Suh, J R. Haywood, G J. Mathews, and N. Q. Lan, 2016 arXiv:1601.01460

[35] B. Allen, W G. Anderson, P R. Brady, D A. Brown, and J D E. Creighton Phys. Rev. D 85 (2012)122006 
[36] L. Blanchet Living Rev. Relativity 5 3, 2002

[37] L. Blanchet Living Rev. Relativity 17 2, 2014

[38] C K. Mishra, K G. Arun and B R. Iyer, Phys. Rev. D 91 (2015) 084040

[39] J R. Wilson and G J Mathews Phys. Rev. Lett. 75 (1995) 4161

[40] J R. Wilson, G J. Mathews, and P. Marronetti Phys. Rev. D 54 (1996) 1317

[41] G J. Mathews, P. Marronetti, and J R. Wilson Phys. Rev. D 58 (1998) 043003

[42] G J. Mathews, and J R. Wilson Phys. Rev. D 61 (2000) 127304

[43] L. Baiotti, T. Damour, B. Giacomazzo, A. Nagar and L. Rezzolla, Phys. Rev. Lett. 105 (2010) 261101

[44] S. Bose, S. Ghosh and P Ajith Class. Quant. Grav 27 (2010) 114001

[45] J S. Read, L. Baiotti, J D E. Creighton, J L. Friedman, B. Giacomazzo, K. Kyutoku, C. Markakis, L. Rezzolla, M. Shibata and K. Taniguchi, Phys. Rev. D 88 (2013) 044042

[46] A. Maselli, L. Gualtieri and V. Ferrari Phys. Rev. D 88 ( 2013) 104040

[47] A. Bauswein and N. Stergioulas Phys. Rev. D, 91, (2015) 124056

[48] A. Bauswein and N. Stergioulas and H-T. Janka Physics of Particles and Nuclei 812 (2015) 24 (2015) 835-838

[49] C. L. Fryer, K. Belczynski, E. Ramirez-Ruiz, S. Rosswog, G. Shen, and A. W. Steiner, Astrophys J. 812 (2015) 24

[50] T. Dietrich, N. Moldenhauer, N K. Johnson-McDaniel, S. Bernuzzi, C M. Markakis, Brügmann B and Tichy W 2015 Phys. Rev. D 92124007

[51] Duez M D, Marronetti P, Shapiro S L and BaumgarteT W 2003 Phys. Rev. D, 67024004

[52] Marronetti P, Duez M D, Shapiro S L and BaumgarteT W 2003 Phys.Rev.Lett. 92, 141101

[53] Miller M, Gressman P, and Suen W-M, 2004 Phys. Rev. D 69064026

[54] Miller M 2005 Phys. Rev. D 71104016

[55] Miller M 2007 Phys. Rev. D 75024001

[56] Uryu K, Limousin F, Friedman J L, Gourgoulhon E and Shibata M 2006 Phys. Rev. Lett. 97171101

[57] Kiuchi K, Sekiguchi Y, Shibata M and Taniguchi K 2009 Phys. Rev. D 80064037

[58] Bernuzzi, Sebastiano; Nagar, Alessandro; Dietrich, Tim; Damour, Thibault 2015 Phys. Rev. Lett. 114161103

[59] De Pietri R, Feo A, Maione F and Löffler F 2015 eprint arXiv:1509.08804

[60] Wilson J R and Mathews G J 2003 Relativistic Numerical Hydrodynamics, (Cambridge University Press, Cambridge, United Kingdom)

[61] Cutler C, Apostolatos T A, Bildsten L, Finn L S, Flanagan E E, D. Kennefik D, Markovic D M, Ori A, Poisson E and Sussman G J, 1993 PRL 702984

[62] Thorne K S 1980 Rev. Mod. Phys. 52299

[63] Arnowitt R, Deser S, and Misner C W 2008 Gen. Rel. Grav. 401997

[64] York J W Jr 1979 Sources of Gravitational Radiation ed Smarr L L (Cambridge University Press, Cambridge, UK) p. 83,

[65] Evans C R 1984 A Method for Numerical Relativity: Simulation of Axisymmetric Gravitational Collapse and Gravitational Radiation Generation., PhD thesis, University of Texas at Austin

[66] E. E. Flanagan, Phys. Rev. Lett., 82 (1999) 1354

[67] J. R. Wilson Sources of Gravitational Radiation ed. L. L. Smarr (Cambridge University Press, Cambridge, UK) p. 423,1979

[68] R. W. Mayle, M. Tavani and J. R. Wilson, Astrophys J. 418 (1993) 398

[69] L. P. Grishchuk Astrophysics Update. Topical and Timely Reviews on Astrophysics, Ed Mason J W (SpringerVerlag and Chichester, UK: Praxis Publishing) p.281, 2004

[70] C. Cutler and E. E. Flanagan, Phys. Rev. D 49 ( 1994) 2658 